\title{
ADSORCIÓN DE PLOMO DIVALENTE DE EFLUENTES METALÚRGICOS EMPLEANDO BENTONITA PILAREADA*
}

\author{
Tinoco Egoavil, Nélida; Fuentes López, Walter² y Bendezú Roca, Yéssica ${ }^{3}$ \\ Facultad de Ingeniería Química de la Universidad Nacional del Centro del Perú

\section{RESUMEN}

Esta investigación fue realizada con los efluentes de la Planta Piloto Metalúrgica de Yauris, con la intención de disminuir los niveles de contaminación con metales pesados, especialmente el plomo, por los daños que ocasiona al ecosistema. En este trabajo se determinó las condiciones óptimas de tiempo, temperatura y pH para la adsorción del plomo. La bentonita natural fue pilareada con una solución de aluminio polihidroxipolimérico proveniente de Mitupampa, distrito de Chongos Alto, provincia de Chupaca, el cual mediante el análisis de Difracción de Rayos $X(D R X)$ presenta un elevado contenido de montmorillonita. Presentaron superficies especificas de $80,52 \mathrm{~m}^{2} / \mathrm{g}$ y $137,023 \mathrm{~m} / \mathrm{g}$, determinada por adsorción de azul de metileno y una capacidad de intercambio catiónico (CIC) de 87,29 y $71,15 \mathrm{meq} / 100 \mathrm{~g}$ de bentonita, respectivamente. Las condiciones óptimas de adsorción se realizaron en un tiempo de remoción de 5 horas, temperatura $30^{\circ} \mathrm{C}$ y pH 5, encontrándose $99,085 \%$ de plomo presente en estado iónico, lográndose un rendimiento de $5,39 \mathrm{~g}$ de $\mathrm{Pb} / \mathrm{g}$ de bentonita pilareada. Al evaluar la remoción del metal en la muestra de efluente metalúrgico se registró un $62,55 \%$, cantidad menor al obtenido con la solución en blanco de $\mathrm{Pb}$, debido a la presencia de otros metales, que actúan como interferentes. Razón por la cual se realizó varias etapas de remoción alcanzando una concentración final de plomo de 0,205ppm, valor que resulta ser menor a los límites máximos permisibles.

Palabras Clave: Montmorillonita, adsorción, remoción, solución pilareante.

\begin{abstract}
The search was developed with effluents from the metallurgical Pilot Plant of Yauris, With the intention of lowering the level of contamination with heavy metals, especially lead, due to the damage, that it caused to the ecosystem. This paper the optimum conditions of time, temperature and $\mathrm{pH}$ were determined for the absorsium of lead the natural betonite was pillared with solution of polihidroxipipo limerick coming from mitupampa, Chongos Alto in the Province of Chupaca, It was analyzed by $x$ - Ray Diffraction (XRD) presenting high content of mont morillomite. The natural and pillared betonites presented specific areas of $80.52 \mathrm{~m}^{2} / \mathrm{g}$ and $137 \mathrm{~m}^{2} /$. That was determined by the absorptions of metlylene and a cation of exchange capacity (CEC) of 87.25 and $71.25 \mathrm{meq} / 100 \mathrm{gr}$. betonite respectively. The optimums conditions took place at a time of removal of 5 hours $30^{\circ} \mathrm{C}$ of temperature and pH5 the percentage of lead inionic state $99,085 \%$ achieving a performance of $5.39 \mathrm{gr} \mathrm{pb} / \mathrm{g}$ of pillared. While evoluting the removal of the metal in the sample of the metallurgical affluent it was registered $62,55 \%$ a quantity lower to the obtained with the solution in white of $\mathrm{Pb}$, caused to the presence of other metals, that act out as interferents. For this reason, we had to carry out several stages of removal reacting a final concentration of lead of $0,265 \mathrm{ppm}$, value that results less to the maximum permissible limits.
\end{abstract}

Key words: Montmorillonite, adsorption, removal, pillared solution.

* Este trabajo de investigación fue recibido el 20/04/2008 retornado para su revisión el 22/09/2008 y aprobado para su publicación el 27/02/2009.

1. E-mail: jolida01@hotmail.com

2. E-mail:wa_fuentes@hotmail.com

3. E-mail: yessiben@yahoo.com 


\section{INTRODUCCIÓN}

La Planta Piloto Metalúrgica de Yauris, se encuentra ubicada al oeste de la ciudad de Huancayo, en el paraje denominado Yauris. Realiza operaciones de concentración de minerales, generando relaves y efluentes con presencia de metales disueltos en concentraciones que superan los límites máximos permisibles. Los relaves son depositados en canchas cercanas a la planta y elimina sus efluentes al río Mantaro, a través de un canal, ocasionando daños al ecosistema. De los metales pesados presentes el plomo divalente es el metal que se encuentra en mayor concentración.

En este sentido, este trabajo de investigación propone el uso de un adsorbente, la bentonita pilareada, para remover el plomo divalente presente en el efluente metalúrgico de la Planta Piloto de Yauris.

La bentonita natural, que en este caso fue extraída de Mitupampa, Chongos Alto, provincia de Chupaca, tiene características adsorbentes muy importantes, para mejorar aun más estas características, se realizó una modificación en su estructura, se colocó pilares entre las hojuelas de arcilla, que le dio mayor estabilidad, obteniéndose de esta forma un adsorbente con mayor área superficial y capacidad de intercambio catiónico.

\section{MATERIAL Y MÉTODOS}

La metodología es experimental. Los métodos analíticos utilizados son los clásicos y los instrumentales (Difracción de rayos $X$, espectrofotometría de absorción atómica, espectrofotometría UV visible).

Realizamos un diseño factorial con 8 experimentos, la variable dependiente fue el porcentaje de remoción de plomo divalente y las variables independientes, tiempo de remoción, temperatura y $\mathrm{pH}$ de la solución a ser adsorbida.

Se extrajo las muestras de bentonita del Yacimiento Beta Mercedes 85 de propiedad de la Cía. Minera Doña Herminia del lugar denominado Mitupampa del distrito de Chongos Alto de la provincia de Chupaca, con la técnica de muestreo tipo $Z$, en un área aproximada de $1000 \mathrm{~m}^{2}$.
El efluente de la Planta Metalúrgica de Yauris fue muestreada de la desembocadura hacia el río Mantaro a 50 metros del centro de proceso, el caudal fue de $6 \mathrm{~L} /$ min, se midieron: temperatura y $\mathrm{pH}$.

\section{Materiales y equipos}

- Agitador magnético LABOR MUSZERIPARI LE 302

- Agitador vibratorio thermolyne AROS 160

- Balanza analítica

- Espectrofotómetro de absorción atómica marca PERKING ELMER modelo 3100 Instituto Geológico Minero Metalúrgico INGEMMET Lima

- Espectrofotómetro UV visible marca SHIMADZU UV 1203

- $\mathrm{pH}$ metro digital marca HACH, modelo EC20

- Cronómetro

- Tamices

- Termómetro

- Materiales de laboratorio

- Acetato de amonio $1 \mathrm{~N}$

- Ácido Fluorhídrico QP

- Agua destilada

- Azul de metileno

- Cloruro de aluminio hexahidratado 0,2 M

- Cloruro de potasio $1 \mathrm{~N}$

- Hidróxido de sodio 0,2 M.

\section{Parte experimental}

El procedimiento seguido fue el siguiente:

\section{Determinación de la composición mineralógica de la} Bentonita:

Pesar 50 gramos de muestra y ponerlo a secar al medio ambiente, moler la muestra y tamizarla (lo mas fino posible). Colocar la muestra fina en un recipiente adecuado del equipo y llevar la muestra al difractómetro de Rayos X.

Se mandó analizar la Bentonita para determinar su composición mineralógica a los laboratorios del INGEMMET, los difractogramas obtenidos nos indican las especies minerales presentes en la muestra, mediante comparación con los patrones conocidos del fichero JCPDS.

\section{Determinación de la composición química}

Pesar una muestra de Bentonita y hacer el ataque con ácido fluorhídrico QP (con mucho cuidado en la campana extractora de gases, por la toxicidad de 
los gases) hasta que no se observe la presencia de partículas. La solución obtenida se diluye, luego se realizan las lecturas de concentración de metales con el equipo de absorción atómica, se obtienen las concentraciones en partes por millón.

Para obtener los componentes en forma de óxidos se multiplica la concentración encontrada por un factor de conversión dependiendo de cada elemento.

\section{Determinación de la superficie específica}

Se hizo mediante el método de adsorción de azul de metileno. Con muestras secas de bentonita (secadas al medio ambiente).

- Moler la muestra y tamizarla (lo más fino posible)

- Pesar 100 miligramos de bentonita, y colocarlo en un matraz de 250 mililitros.

- Preparar soluciones de azul de metileno a concentraciones de: 0,$900 ; 0,800 ; 0,700 ; 0,600$; 0,$550 ; 0,500 ; 0,450 ; 0,400 ; 0,350 ; 0,300 ; 0,250$; 0,$200 ; 0,100$, ( g/L ). (Para estas soluciones se utilizó azul de metileno Merck extra, para microscopia, con un peso molecular de 379,90 $\mathrm{g} / \mathrm{mol}$.).

- Agregar 100 mililitros de cada solución a diferentes matraces que contienen los 100 miligramos de bentonita.

- Tapar las muestras y agitarlas durante 12 horas y reposar por 24 horas.

- Tomar muestras de la solución sobrenadante, para el análisis por espectrofotometría UV visible.

\section{Determinación de la capacidad de intercambio catiónico}

Se determina la concentración de 4 iones presentes en la muestra, los cuales se realizan por separado.

\section{a) Sodio y potasio}

- Pesar 2,5 gramos de muestra, lavar con agua destilada de 10 en $10 \mathrm{~mL} 5$ veces (colocado en un papel filtro).

- Agregar $50 \mathrm{~mL}$ de solución de acetato de amonio1 $\mathrm{N}$ con un $\mathrm{pH} 7$ (de 10 en $10 \mathrm{~mL}$ ) y filtrar.

- Agitar bien la muestra resultante, y llevarlo a leer en el espectrofotómetro de absorción atómica.

\section{b) Calcio y magnesio:}

- Pesar 2,5 gramos de muestra, lavar con agua destilada de 10 en $10 \mathrm{~mL} 5$ veces (colocado en un papel filtro).
- Agregar $50 \mathrm{~mL}$ de solución de cloruro de potasio 1 $\mathrm{N}$ con un pH 7 (de 10 en $10 \mathrm{~mL}$ ) y filtrar.

- Agitar bien la muestra resultante, y llevarlo a leer en el espectrofotómetro de absorción atómica.

Como resultado de este análisis se tendrán la concentración de cada metal en partes por millón. Los cuales se multiplican por un factor de trabajo para obtener las miliequivalentes de cada elemento.

Cuya unidad será en me / $100 \mathrm{~g}$ de bentonita, que representa el intercambio total de iones presentes en la muestra de bentonita.

\section{Pilareado de la bentonita}

Para este caso se usaron los procedimientos propuestos y los parámetros encontrados por Bendezú \& Bullón, 2002.

\section{Preparación de la muestra}

Para la operación de pilareado se necesita una bentonita purificada, y para ello se realizó por el método de sedimentación, el cual consiste en:

- Pesar 50 gramos de muestra de bentonita y colocarlo en una probeta de 1 litro, adicionar 700 mililitros de agua destilada y agitar vigorosamente por algunos minutos, luego aforar y dejar en reposos por un lapso de 10 minutos.

- Extraer la solución sobrenadante hasta una profundidad de $10 \mathrm{~cm}$, desechar el resto por centrifugación, separar la mayor parte de líquido de la solución.

- La muestra obtenida de bentonita secarla a $100^{\circ} \mathrm{C}$ por un tiempo de 24 horas.

- Repetir todos los pasos, hasta obtener la cantidad necesaria de bentonita purificada para el pilareo.

\section{Preparación de la solución pilareante \\ (Al13 polihidroxipolimérico)}

- Preparar soluciones de $\mathrm{NaOH}$ y $\mathrm{AlCl}_{3} \cdot 6 \mathrm{H}_{2} \mathrm{O}$, ambas a concentración de 0,2 molar.

- A una solución agitada de $\mathrm{AlCl}_{3} \cdot 6 \mathrm{H}_{2} \mathrm{O}$, adicionar $\mathrm{NaOH}$ con una velocidad de $1 \mathrm{~mL} / \mathrm{min}$, hasta obtener una relación en volumen de $\mathrm{OH} / \mathrm{Al}$ igual a 2,5 .

- Envejecer la solución preparada, por un día a temperatura ambiente, esto en constante agitación, hasta que la solución quede clara y no haya presencia de precipitado. 


\section{Pilareado de la bentonita con aluminio polihidroxipolimérico}

- Preparar una suspensión acuosa al $2 \%$ en peso con la bentonita purificada.

- Añadir esta suspensión a la solución pilareante preparada anteriormente, todo en constante agitación, hasta obtener una relación final de Al / bentonita igual a $2 \mathrm{mmol} / \mathrm{g}$ bentonita.

- Mantener la muestra a un pH entre 3 y 6, y dejar reaccionar por 12 horas, luego que transcurre el tiempo, el producto obtenido fue centrifugado $\mathrm{y}$ lavado con agua destilada (unas 5 veces)

- La bentonita obtenida (coagulada) se seca a $100^{\circ} \mathrm{C}$ por 24 horas.

- La muestra seca obtenida se lleva a calcinación por 4 horas a temperatura de $300^{\circ} \mathrm{C}$.

\section{Remoción de plomo de la muestra de efluente metalúrgico}

- Pesar 0,4 gramos de bentonita pilareada y colocarla en un matraz de $250 \mathrm{ml}$.

- Agregar $100 \mathrm{ml}$ de efluente al matraz, llevar el pH a 5 , temperatura a $30^{\circ} \mathrm{C}$.

- Tapar y agitar por 5 horas a $110 \mathrm{rpm}$ aprox.

- Filtrar.

- La muestra obtenida se analiza con ayuda del equipo de espectrofotometría de absorción atómica, para determinar la concentración final de plomo.

- Realizar etapas de remoción hasta llegar a la concentración permisible de acuerdo al Ministerio de Energía y Minas.

\section{RESULTADOS}

\section{Composición mineralógica}

Análisis de difracción de Rayos X (DRX) por el método de polvos de la muestra de bentonita proveniente de Chongos Alto de la Región Junín.

Tabla 1. Composición mineralógica bentonita natural.

\begin{tabular}{ccc}
\hline Mineral & Fórmula & Porcentaje (\%) \\
\hline Montmorillonita & $\mathrm{Ca}_{02}(\mathrm{Al}, \mathrm{Mg})_{2} \mathrm{Si}_{4} \mathrm{O}_{10}(\mathrm{OH})_{24} \mathrm{H}_{2} \mathrm{O}$ & 86,69 \\
Cuarzo & $\mathrm{SiO}_{2}$ & 7,86 \\
Albita & $(\mathrm{Na}, \mathrm{Ca})(\mathrm{Si}, \mathrm{Al})_{4} \mathrm{O}_{8}$ & 4,39 \\
Muscovita & $(\mathrm{K}, \mathrm{Na})(\mathrm{Al}, \mathrm{Mg}, \mathrm{Fe})_{2}$ & 1,06 \\
\hline
\end{tabular}

Fuente: Laboratorio de DRX, INGEMMET.

\section{Composición química}

Análisis de fluorescencia de Rayos X (FRX) de la bentonita natural en el Laboratorio de Química Analítica del Instituto Geológico Minero Metalúrgico.

Tabla 2. Composición química de la bentonita natural.

\begin{tabular}{cc}
\hline Óxidos & Porcentaje (\%) \\
\hline $\mathrm{SiO}_{2}$ & 48,3 \\
$\mathrm{Al}_{2} \mathrm{O}_{3}$ & 17,9 \\
$\mathrm{TiO}_{2}$ & 0,15 \\
$\mathrm{MnO}$ & 0,02 \\
$\mathrm{Fe}_{2} \mathrm{O}_{3}$ & 2,52 \\
$\mathrm{CaO}$ & 2,11 \\
$\mathrm{MgO}$ & 2,81 \\
$\mathrm{~K}_{2} \mathrm{O}$ & 0,15 \\
$\mathrm{Na}_{2} \mathrm{O}$ & 0,08 \\
$\mathrm{PxC}$ & 7,94
\end{tabular}

Fuente: Laboratorio de Química, INGEMMET.

Tabla 3. Distancia interplanar alcanzada de la bentonita pilareada.

\begin{tabular}{cc}
\hline 2 Theta (deg) & d (A) \\
\hline 5,60 & 15,75 \\
\hline
\end{tabular}

Fuente: Laboratorio de Difracción INGEMMET

\section{Superficie específica.}

Análisis de espectrofotometría UV-Visible.

Tabla 4. Concentración de azul de metileno después del contacto con la bentonita pilareada.

\begin{tabular}{cc}
\hline $\begin{array}{c}\text { Concentración } \\
\text { Inicial (g/L) }\end{array}$ & $\begin{array}{c}\text { Concentración } \\
\text { Final } \mathbf{( g / L )}\end{array}$ \\
\hline 0,900 & 0,329 \\
0,700 & 0,248 \\
0,600 & 0,205 \\
0,550 & 0,183 \\
0,500 & 0,171 \\
0,450 & 0,160 \\
0,400 & 0,130 \\
0,350 & 0,098 \\
0,300 & 0,072 \\
0,250 & 0,050 \\
0,200 & 0,028 \\
0,100 & 0,004 \\
\hline
\end{tabular}

Fuente: Laboratorio de Espectrofotometría UV-Visible, FIQUNCP. 
Con estos resultados se determina su isoterma y se calcula la superficie específica que fue de $134,01 \mathrm{~m}^{2} / \mathrm{g}$ de bentonita.

\section{Capacidad de intercambio catiónico}

Tabla 5: Capacidad de intercambio catiónico de la bentonita pilareada.

\begin{tabular}{cc}
\hline Iones cambiables & $\mathrm{CIC}(\mathbf{C m o l} / \mathbf{k g})$ \\
\hline $\mathrm{Ca}^{++}$ & 62,5 \\
$\mathrm{Mg}^{++}$ & 7,66 \\
$\mathrm{Na}^{+}$ & 0,18 \\
$\mathrm{~K}^{+}$ & 0,81 \\
Total & 71,15 \\
\hline
\end{tabular}

Fuente: Laboratorio de Suelos de UNALM.

\section{Plomo en el efluente metalúrgico}

Tabla 6. Concentración de plomo en la muestra de efluente metalúrgico.

\begin{tabular}{ccc}
\hline Metal & $\mathbf{m g} / \mathbf{L}$ & $\mathbf{p p m}$ \\
\hline Plomo & 21,1 & 21,1 \\
\hline
\end{tabular}

Fuente: Laboratorio de Química INGEMMET.

\section{Porcentaje de remoción de plomo en la muestra de efluente metalúrgico}

Tabla 7. Porcentaje de remoción de plomo en la muestra de efluente metalúrgico.

\begin{tabular}{ccc}
\hline $\begin{array}{c}\text { Concentración } \\
\text { inicial (ppm) }\end{array}$ & $\begin{array}{c}\text { Concentración } \\
\text { final (ppm) }\end{array}$ & \% de remoción \\
\hline 21,1 & 7,9 & 62,55 \\
\hline
\end{tabular}

Fuente: Elaboración propia.

\section{Rendimiento de la bentonita pilareada en la remoción de plomo de la muestra de efluente metalúrgico}

Tabla 8. Rendimiento de la bentonita pilareada en la remoción de plomo de la muestra de efluente metalúrgico.

\begin{tabular}{cccc}
\hline $\begin{array}{c}\text { Peso Inicial } \\
\text { de } \mathrm{Pb}, \mathbf{g}\end{array}$ & $\begin{array}{c}\text { Peso final } \\
\text { de } \mathrm{Pb}, \mathbf{g}\end{array}$ & $\begin{array}{c}\text { Peso adsorbido } \\
\text { de } \mathrm{Pb}, \mathbf{g}\end{array}$ & $\begin{array}{c}\% \\
\text { Rendimiento }\end{array}$ \\
\hline 0,0211 & 0,0079 & 0,0132 & 3,3 \\
\hline
\end{tabular}

Fuente: Elaboración propia.
Etapas de remoción de plomo en el efluente metalúrgico

Tabla 9. Concentración de plomo en cada etapa de remoción.

\begin{tabular}{cc}
\hline Etapas de remoción & $\begin{array}{c}\text { Concentración final } \\
\text { de } \mathrm{Pb}(\mathbf{p p m})\end{array}$ \\
\hline Primera & 7,90 \\
Segunda & 5,20 \\
Tercera & 3,20 \\
Cuarta & 0,21 \\
\hline
\end{tabular}

Laboratorio de Análisis Instrumental.

\section{DISCUSIÓN}

En cuanto a la composición mineralógica de la muestra de bentonita proveniente de Chongos Alto, después de realizar el análisis de DRX por el método de polvos, se puede apreciar que el mineral arcilloso que se encuentra en mayor cantidad, $86,69 \%$, es la montmorillonita cálcica, el cual nos confirma la buena calidad de la bentonita. Los otros minerales que vienen a ser impurezas, representan el $13,31 \%$ del total, que no significa ningún peligro o interferencia en el proceso de pilareado.

Realizando una comparación entre la bentonita natural y pilareada, en cuanto a la distancia interplanar y la superficie específica, se nota que el de la bentonita pilareada presenta valores más elevados en ambos casos. Esto se debe principalmente a la presencia de los pilares fijos de aluminio entre las láminas de la arcilla tratada. Este aumento en la superficie específica le confiere a este material mejores características adsorbentes, que actuarán en la remoción de plomo ionico.

La disminución de la $\mathrm{CIC}$ en el caso de la bentonita pilareada (de 87,23 a $71,15 \mathrm{me} / 100 \mathrm{~g}$ de bentonita) se debe a la presencia del ion Al13, con una valencia +7 , el cual al estar en contacto con la bentonita intercambia los iones calcio, magnesio, sodio y potasio, reduciendo de esta manera la presencia de estos cationes.

En la remoción de plomo con la bentonita pilareada, se trabajó con efluente metalúrgico con una concentración inicial de plomo de 21,1ppm, el cual después de su tratamiento, 5 horas de tiempo de contacto, $30^{\circ} \mathrm{C}$ y 
un pH de 5, arrojó como concentración final 7,9ppm, obteniéndose un porcentaje de remoción de 62,55 \%, el cual nos demuestra que la bentonita pilareada posee un gran poder adsorbente. Sin embargo este resultado no es el esperado, en cuanto al cumplimiento de los Límites Máximos Permisibles (LMPs) según el Ministerio de Energía y Minas que es de 0,4ppm de plomo. Para lograr este resultado, se realizó pruebas de adsorción sucesivas, en 4 etapas, llegando a obtener en la cuarta etapa una concentración de plomo de 0,205ppm, pudiéndose de esta manera lograr el objetivo principal de este tema de investigación.

\section{CONCLUSIONES}

- El análisis mineralógico de la muestra de bentonita proveniente de Chongos Alto-Junín, mediante difracción de Rayos X por el método de polvos, muestra que el mineral arcilloso que se encuentra en mayor cantidad, $86,69 \%$, es la montmorillonita cálcica, el cual nos confirma la buena calidad de la bentonita. Los otros minerales que vienen a ser impurezas, representan el $13,31 \%$ del total, que no significa ningún peligro o interferencia en el proceso de pilareado.

- La bentonita pilareada presentó una distancia interplanar de 15,75 A, superficie específica de 134,01 $\mathrm{m}^{2} / \mathrm{g}$ y una capacidad de intercambio catiónico de $71,15 \mathrm{me} / 100 \mathrm{~g}$ de bentonita.

- La concentración de plomo en la muestra de efluente metalúrgico es de $21,1 \mathrm{ppm}$, valor que representa el $0,0021 \%$ en peso.

- Con las condiciones de tiempo de contacto de 5 horas, $30^{\circ} \mathrm{C}$ de temperatura de remoción y un $\mathrm{pH}$ de 5 , después de 4 etapas sucesivas de tratamiento con bentonita pilareada como adsorbente, se removió el 99,10\% de plomo presente en estado iónico, lográndose un rendimiento de 5,39 g. de plomo/g. de bentonita pilareada.

- Se removió el plomo hasta 0,2054 ppm, valor que ese encuentra dentro de los límites máximos permisibles (LMPs) estipulados por el Ministerio de Energía y Minas, que es de 0,4 ppm de plomo.

\section{LITERATURA CITADA}

García, R. E.; y Suárez, B. M. 2000. Las Arcillas: Propiedades Usos, Universidad de Salamanca.

Domínguez, J. M. y Schifter, I. 1999. Las Arcillas: “EI Barro Noble", La Ingeniería Molecular.

Gonzales, G. S. \& Martín V. J. 1999. Caracterización y Propiedades de una Bentonita de Almería", Anales de la Real Sociedad de Física y Química 52 pp. 339 - 346.

Jiménez, S. J. \& Justo A. L. 1985. Geotécnica y Cimientos. Vol. I. Propiedades de los Suelos y de las Rocas., segunda Edición. Editorial Rueda, $466 \mathrm{pp}$.

Sun Kou, M. R.; Sandoval, J.; \& Terrones, C. 2000; Boletin de la Sociedad Química del Perú, vol LXVI, No 4, diciembre del 2000 García, R. E.; Suárez B. M., Las Arcillas: Propiedades Usos, Universidad de Salamanca.

Odriozola, V., Situación y opciones para la cuenca más negra de la Argentina, Matanza Riachuelo.

Contaminación con plomo, Centro de Información de agua, Estados Unidos

Niveles Máximos Permisibles para Efluentes Minero - Metalúrgicos

http://www.minem.gob.pe/archivos/guias/rm 011-96.pdf.

Sun K., M. R. \& Sandoval, J. 2004. Desarrollo de Adsorbentes para la Remoción de Metales de Efluentes Industriales. Proyecto V.G.-CYTED, en prensa.

Pinzón, B. J. A. 2002. Superficie específica de una bentonita mediante la adsorción de azul de metileno, departamento de Química, Facultad de Ciencias, Universidad Nacional de Colombia. 